\title{
Thoracic ultrasound experiences among respiratory specialty trainees in the UK
}

\author{
Authors: Parthipan Sivakumar, ${ }^{A, *}$ Meera Kamalanathan, ${ }^{A, *}$ Anne S Collett ${ }^{B}$ and Liju Ahmed ${ }^{B}$
}

\begin{abstract}
Achieving competence in thoracic ultrasound is a mandatory requirement for the successful completion of respiratory specialty training in the UK. We evaluated trainee competencies, access to training and confidence in thoracic ultrasound by means of a nationally distributed survey with the participation of 202 (of approximately 600 ) respiratory trainees. $65.8 \%$ (131/199) of responders are RCR Level 1 accredited and $20.6 \%$ $(22 / 107)$ of these trainees had performed fewer than 20 ultrasounds in the past year. $29.2 \%$ (50/171) of trainees reported that access to an ultrasonographer for advice was either 'not easy' or 'impossible'. 59\% (107/171) of all respondents are 'never' or 'rarely' supervised, with $60 \%$ (102/169) of queries answered by real-time evaluation or review of stored media. Encouragingly ultrasound training has evolved considerably in recent years, but ongoing work needs to focus on improving supervision and training. There is a case for reviewing current guidance and to consider tailoring training and expectations to align with the specific needs of respiratory registrars. We propose a revision of the current Royal College of Radiologists framework towards a respiratory specialist led accreditation in thoracic ultrasound.
\end{abstract}

KEYWORDS: Education, pleural, respiratory, training, ultrasound

\section{Introduction}

Respiratory physicians are increasingly using thoracic ultrasound following a National Patient Safety Agency recommendation ${ }^{1}$ published in 2008 to use ultrasound during pleural procedures to improve success rates and reduce complications. This also features in the current British Thoracic Society (BTS) pleural disease guideline. ${ }^{2}$ In a bid to formalise ultrasound skills training for medical specialties, the Royal College of Radiologists (RCR) published its Ultrasound training recommendations for medical and surgical specialties in 2005. This has subsequently been updated ${ }^{3}$ and remains the key recommendation for all medical and surgical specialties who wish to use ultrasound within their specialty to the same level as a trained radiologist.

Authors: A specialty trainee, St Thomas' Hospital, London,


contributed equally
The RCR also recognised the use of ultrasound in non-radiology settings, notably when being used by other specialties to answer more focused clinical questions. A second document, Focused ultrasound training standards $s^{4}$ was published, which is aimed at training targeted ultrasound to answer a specific clinical question or to direct procedures. With respect to respiratory medicine, this refers to the ultrasound-guided drainage of pleural effusions.

Both documents set out a knowledge base and guidelines for training and practice in order to gain Level 1, then Level 2 competency, with additional guidance on maintaining these skills. There are subtle but important differences between the two guidelines; the major difference being the training period required to achieve Level 1 competency. However, the knowledge base and competencies are broadly the same (Table 1). The guidelines have been adopted by the Joint Royal Colleges of Physicians Training Board (JRCPTB) with focused Level 1 competence a mandatory curriculum requirement for respiratory trainees. ${ }^{5}$

We sought to evaluate competencies, access to training and confidence in undertaking thoracic ultrasound among respiratory registrars in the UK.

\section{Methods}

We formulated a 22-question online survey incorporating fivepoint Likert scales to assess factors pertaining to experience and confidence. The survey (www.tinyurl.com/ultrasoundsurvey) was circulated via the relevant JRCPTB specialist advisory committee to all training programme directors for dissemination to trainees in the UK. Responses were collected between October 2015 and June 2016. No regional ethics approval was required and participation was entirely voluntary with responses anonymised.

\section{Results}

\section{Demographics}

In total, 202 (of approximately 600) respiratory trainees from 14 deaneries completed the survey. The results are summarised in Table 2. Not all responders answered every question.

\section{Competencies}

Of 199 responders, 131 (65.8\%) had achieved Level 1 competence in thoracic ultrasound and 20.6\% (22/107) of Level 1 accredited trainees had performed fewer than 20 ultrasounds in the previous year. 
Table 1. A summary of the current Royal College of Radiologists (RCR) guidelines. Adapted from Ultrasound training recommendations for medical and surgical specialties and Focused ultrasound training standards. 3,4

\section{Level 1. Training and practice}

$>$ Full RCR guideline: At least one session per week over a period of no less than 3 months with approximately five scans per session (under supervision of an experienced practitioner)

> 'Focused' RCR guideline: Observe 20 thoracic ultrasound examinations, perform 20 examinations on normal patients, perform 20 examinations on patients with pleural effusion, perform 20 thoracocenteses or drain placements using both guided and non-guided techniques

$>$ A logbook should be kept

$>$ Supervision by Level 2 practitioner or by a Level 1 practitioner with at least 2 years' experience

$>$ Attendance at a theoretical course with support from textbooks and literature

\section{Level 1. Competencies to be acquired}

$>$ Recognition of normal anatomy (pleura, diaphragm, heart, liver, spleen)

> Recognition of pathology (pleural effusion, pleural thickening, consolidated lung)

$>$ Estimated depth of pleural effusion

$>$ Use of colour flow Doppler

Guided thoracocentesis and drain placement

\section{Level 2. Knowledge base}

$>$ As for Level 1, with more detailed understanding of pleural disease

\section{Level 2. Training and practice}

$>$ At least 1 year of experience at Level 1 with a minimum of two examinations performed per week

$>$ A further 100 examinations to encompass the full range of conditions and procedures

$>$ Supervision of training by a Level 2 practitioner with at least 2 years' experience

\section{Level 2. Competencies to be acquired}

$>$ As for Level 1 with additional advanced use of Doppler ultrasound $>$ Ultrasound-guided lung and nodal biopsy

\section{Maintenance of skills: all levels}

$>$ CPD and maintenance of practical skills

> Perform at least 20 ultrasound examinations per year

> Regular meetings with radiological colleagues

> Named radiologist as an 'ultrasound mentor'

\section{Access to training}

In total, 59\% (107/171) of responders reported that they are 'never' or 'rarely' supervised performing thoracic ultrasound and $27.4 \%$ (48/175) stated that radiologists at their current trust were not willing to provide training. Access to an ultrasonographer for advice was reported as either 'not easy' or 'impossible' by $29.2 \%(50 / 171)$ of responders.

A total of $9 \%(15 / 167)$ of responders reported that there were no Level 1 or Level 2 accredited respiratory consultants at their current hospital.

\section{Confidence and practices}

Confidence in identifying thoracic pathology on ultrasound among Level 1 accredited trainees is illustrated in Fig 1. Of 107 respondents, 25 (23.4\%) reported 'rarely' or 'never' using thoracic ultrasound to perform pleural procedures (excluding aspiration of a pneumothorax). 90.1\% (155/172) were 'often' or 'always' confident in performing pleural procedures based on their thoracic ultrasound assessment.

\section{Discussion}

There has been considerable progress in delivering ultrasound training following its relatively recent introduction into the respiratory curriculum. Although RCR guidelines have provided this framework and the majority of trainees are signed off as Level 1, a fifth of Level 1 accredited responders are not fulfilling the minimum requirement of 20 ultrasounds a year to maintain their competencies. It is unclear why this is, but reasons may include deferral of bedside scans to radiology either because of a lack of experienced consultant ultrasound practitioners to provide supervision or limited access to an ultrasound machine. Another possibility is a lack of trainee awareness of the two RCR guidelines and the requirements to maintain their competencies. Furthermore, confidence among accredited trainees in identifying pathology beyond pleural effusions and lung consolidation is inconsistent, which may reflect variation in training experiences.

These data also examine current access to supervision. There is variation in the confidence of trainees and the skill of their supervisors, as well as limited access to radiologists for training. The reasons for the reluctance of radiologists to train respiratory registrars are unclear from this survey but may relate to commitments to their own trainees. With the increasing assimilation of thoracic ultrasound into the respiratory domain, respiratory consultants may be required to provide supervision despite a lack of formal training in thoracic ultrasound. Access to quality supervision may be improved by ensuring trainees rotate through a specialist pleural service in the early stages of their specialist career.

A considerable proportion of trainees communicate their queries by verbal description, the reliability of which is highly dependent on operator experience. This is in stark contrast to other diagnostic radiological tests, such as chest $\mathrm{X}$-ray and computerised tomography, which are directly reviewed by senior clinicians with specialist radiology input sought in challenging cases. Surprisingly, we identified a small group of trainees who do not always use ultrasound for pleural procedures, contrary to BTS guidelines. The 
Table 2. Summary of responses to survey questions

Responder demographics and survey
questions concerning competency, access to
training, confidence and practices
Training grade ( $n=202$ )
ST3-ST4
ST5-ST6
ST7
Post CCT
Level 1 competence ( $n=199$ )
Training grade if Level 1 competent ( $n=131$ )
ST3-ST4
ST5-ST6
ST7
Post CCT
Deemed Level 1 competent by: ( $n=108$ )
Radiologist
Respiratory consultant (unsure of experience)
Respiratory consultant with Level 1 competence
Respiratory consultant with Level 2 competence

Number of ultrasound examinations performed in the past year by Level 1 accredited trainees $(n=107)$

0

$1-20$

$21-50$

Over 50

At your current hospital, are radiologists willing to train respiratory trainees in thoracic ultrasound? $(n=175)$

Don't know/haven't asked

No

Yes

$94(53.7 \%)$

$48(27.4 \%)$

$33(18.9 \%)$

If unsure about findings, how easy is it to seek advice from a competent ultrasonographer? $(n=171)$

Impossible

$5(2.9 \%)$

Not easy

$45(26.3 \%)$

Neutral

Easy

Very easy

How are ultrasound queries usually reviewed? $(n=169)$

Real-time ultrasound evaluation

Review of captured images/videos

You provide a verbal description of findings
Table 2. Summary of responses to survey questions

\section{Responder demographics and survey questions concerning competency, access to training, confidence and practices} n (\%)

When supervised, how often do you feel your supervisor is adequately trained to do so? $(n=162)$

$\begin{array}{ll}\text { Never } & 9(5.6 \%) \\ \text { Rarely } & 4(2.5 \%) \\ \text { Sometimes } & 20(12.3 \%) \\ \text { Often } & 51(31.5 \%) \\ \text { Always } & 78(48.1 \%)\end{array}$

Do you use ultrasound for pleural procedures (other than aspiration of a pneumothorax)? $(n=169)$

Never

Rarely

Sometimes

Often

Always

$107(63.3 \%)$

How often do you feel confident performing pleural procedures based on your own assessment of the thorax on ultrasound? $(n=172)$

\begin{tabular}{ll} 
Never & $1(0.6 \%)$ \\
Rarely & $2(1.2 \%)$ \\
Sometimes & $14(8.1 \%)$ \\
Often & $80(46.5 \%)$ \\
Always & $75(43.6 \%)$ \\
\hline CT = certificate of completion of training; ST = specialty training year
\end{tabular}

reasons for this are unclear, and we cannot exclude factors such as misinterpretation of the question or limited access to ultrasound equipment. $30 \%$ of trainees consider themselves confident enough to diagnose a pneumothorax, which is a surprising finding given the complexity of using ultrasound for pneumothorax especially in patients with bullous emphysema. However, this may reflect the front-line role of specialty trainees, where ultrasound assessments are conducted in the context of a patient's medical history and pre-existing imaging. We acknowledge that we are unable to reliably determine which guideline is satisfied when respondents state they are Level 1 competent (a focused competency versus a full Level 1 competency). We also recognise that with responses from only $30 \%$ of UK trainees, there is potential for responder as well as recall bias to confound the data, which may select for more 'negative' responses. However, this effect is likely to be small as a significant proportion of respondents are senior trainees who have achieved their curriculum goals.

The broader question is whether the current RCR framework is suited to respiratory trainees. We believe that the curriculum and training delivery should be revised to better align with the specific needs and scope of practice of respiratory trainees. If ultrasound exposure is limited in real-world practice, we 


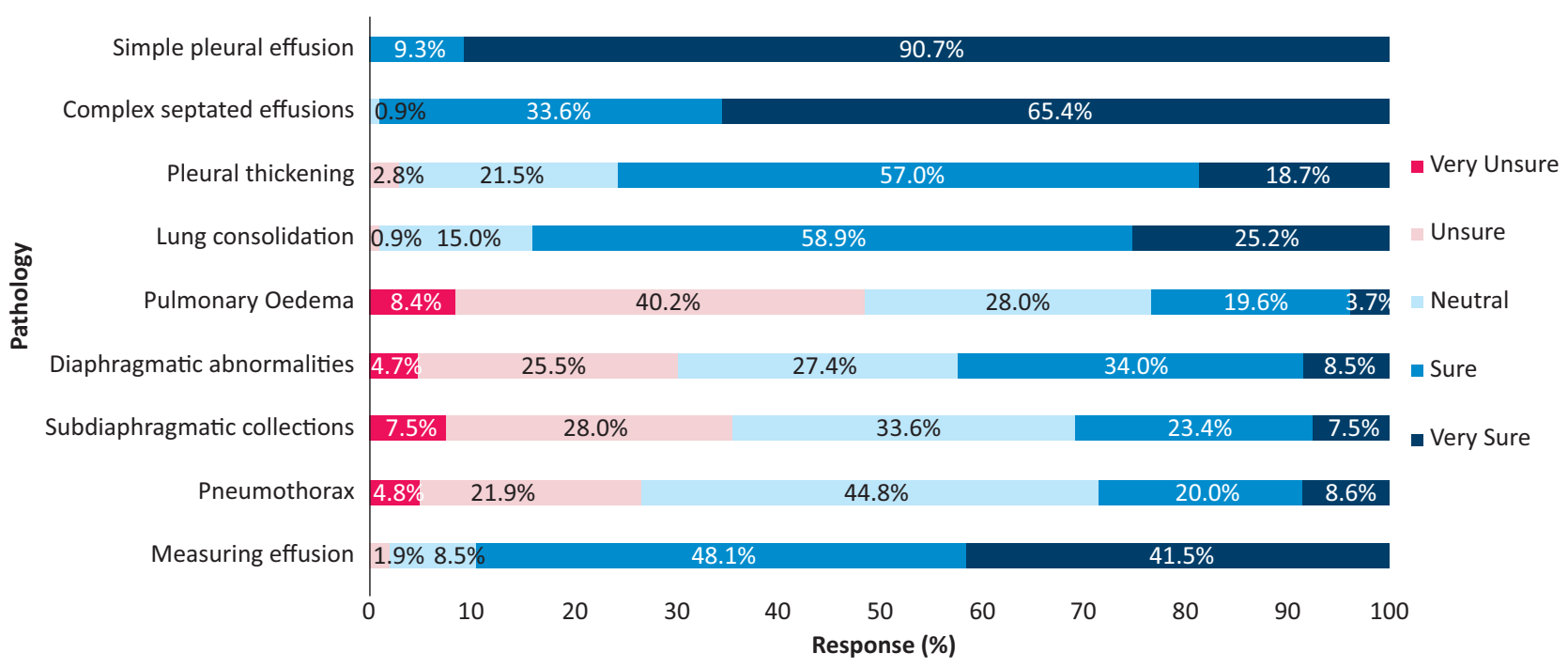

Fig 1. Confidence in identifying thoracic pathology among Level 1 accredited trainees. $n=107$.

should also consider other educational adjuncts, for example simulation training for both ultrasound evaluation and procedures, as well as the development of a wiki (collaborative open-access online repository) populated with ultrasound video case studies for use by both specialty registrars and consultants to develop and maintain their skills.

Rather than a unilateral approach to Level 1 competency sign off, perhaps we should aim for a more vertical progression, with competencies being developed throughout training. This could include defining minimum competency requirements for each stage of training from ST3 (specialty trainee year 3) to ST7 (specialty trainee year 7), thus ensuring standardised training with an achievable goal at every stage. This will allow realistic training and assessment opportunities to be set for trainees during different placements. This should promote safe patient care as it will enable referral onwards to a pleural specialist or radiologist when the limit of competence is reached. Similar to the model of echocardiographic training adopted by our cardiology colleagues, ${ }^{6}$ this form of ultrasound training could culminate in a formal examination and accreditation for those who wish to subspecialise in pleural disease. Though the format of this assessment should be tailored to curriculum requirements of UK trainees, we can certainly seek the advice of our colleagues in Denmark and Australia who already lead the way in the development of validated assessment tools. ${ }^{7,8}$

As current trainees become respiratory consultants, the curriculum and method of training needs to be revised to ensure that these future consultants have the experience and skills required to supervise and train the next generation of respiratory registrars. We believe that a robustly structured ultrasound training scheme with a formal accreditation can help to achieve this. To attain all of this on a national scale, constructive collaboration between pleural specialists in the newly formed BTS pleural specialist advisory group, the JRCPTB specialist advisory committee and trainees will be essential.

\section{Conflicts of interest}

The authors have no conflicts of interest to declare.

\section{Author contributions}

PS and MK are joint first authors who contributed equally to this paper. They conceived and designed the study, collected the data, performed the data analysis and wrote the manuscript. AC and LA contributed to study conception, critical revisions and approved the final version for publication. All authors are accountable for all aspects of the work.

\section{References}

1 Agency National Patient Safety. Chest drains: risks associated with the insertion of chest drains. Rapid Response Report NPSA/2008/RRR003. London: NPSA, 2008. www.nrls.npsa.nhs.uk/ resources/?entryid45=59887 [Accessed 5 July 2017].

2 Maskell N, British Thoracic Society Pleural Disease Guideline Group. British Thoracic Society Pleural Disease Guidelines-2010 update. Thorax 2010;65:667-9.

3 The Royal College of Radiologists. Ultrasound training recommendations for medical and surgical specialties, 2nd edn. London: RCR, 2012.

4 The Royal College of Radiologists. Focused ultrasound training standards. London: RCR, 2012.

5 Joint Royal Colleges of Physicians Training Board. Specialty training curriculum for respiratory medicine August 2010 (Amendments May 2014). London: JRCPTB, 2015.

6 British Society of Echocardiography. Accreditation in adult TTE echocardiography: Information pack. London: BSE, 2016.

7 Skaarup SH, Laursen CB, Bjerrum AS, Hilberg O. Objective and structured assessment of lung ultrasound competence. A multispecialty Delphi consensus and construct validity study. Ann Am Thorac Soc 2017; 14:555-60.

8 Williamson JP, Twaddell SH, Lee YC et al. Thoracic ultrasound recognition of competence: a position paper of the Thoracic Society of Australia and New Zealand. Respirology 2017;22:405-8.

Address for correspondence: Dr Parthipan Sivakumar, Department of Thoracic Medicine, St Thomas' Hospital, Westminster Bridge Road, London SE1 7EH, UK. Email: deepan.sivakumar@kcl.ac.uk 